\title{
Meta-analyses based on summary data can provide timely, thorough and reliable evidence: don't dismiss them yet
}

To the Editor - The inclusion of fraudulent trials in evidence synthesis may lead to unreliable or biased results, so there have been calls to use individual participant data (IPD) exclusively when conducting meta-analyses of COVID-19 studies ${ }^{1}$.

Certainly, with multiple high-profile cases of fraudulent trials in recent years, the collection of IPD would ensure that such trials are unearthed, but the time needed to collect and process IPD precludes rapid evidence synthesis. We argue that a prospective and collaborative approach to meta-analysis of summary data can provide timely, thorough and robust evidence synthesis, and an extra level of scrutiny of trial results.

The work by Lawrence and colleagues highlights important challenges for evidence synthesis exposed by the COVID-19 pandemic ${ }^{1}$. As they diligently point out, fraudulent research included in numerous meta-analyses has led to considerable support for the use of an anti-parasitic drug, ivermectin, in the prevention and treatment of COVID-19. Although the issue of fraudulent or fabricated studies is not new or specific to COVID-19, the pandemic has expedited the immediate release of trial results through press releases or pre-prints ${ }^{2}$, with multiple high-profile retractions ${ }^{3}$. Increasing the visibility of these issues is important, and a call for a change in the way the scientific community approaches evidence synthesis is warranted. However, as meta-analysis researchers, we disagree that all "meta-analyses based on summary data alone are inherently unreliable" ${ }^{1}$.

Systematic reviews and meta-analyses are commonly planned retrospectively, after eligible trials have reported their results, which can introduce bias into both review methods and analysis methods ${ }^{4}$. These reviews typically use summary data extracted from trial publications or other reports, and may overlook unpublished and ongoing trials ${ }^{5}$. Such summary data meta-analyses have the potential to be unreliable, and we agree that they risk including fraudulent trials, exacerbating this situation further. Meta-analyses of the effects of ivermectin for COVID-19 provide a good example of how standard approaches to evidence synthesis can lead to ungrounded claims of treatment benefit.
A prospective and collaborative approach to meta-analysis (prospective meta-analysis (PMA)) of summary data, wherein methods are planned before results of included trials are known ${ }^{4}$, is a viable alternative. Working with investigators can provide access to more-detailed, standardized trial results, thereby reducing reporting and other data-availability biases. PMA can also improve the breadth of analyses, enabling more-nuanced meta-analysis results, such as whether treatment effects vary by participant characteristics. Importantly, investigators provide results directly to the review team, allowing additional scrutiny and less room for fabrication of data. In a sense, collaborative PMA of summary data brings advantages akin to those associated with IPD meta-analysis ${ }^{6}$. One such PMA approach, FAME ${ }^{4}$, also involves prospectively monitoring how evidence from trials is accumulating, in order to anticipate the earliest opportunity for a potentially definitive meta-analysis, potentially months or years before all study results are available. Hence, PMA of summary data can provide more timely and less biased evaluations of treatment effects than standard approaches can ${ }^{4}$.

An example is our recent collaborative PMA investigating the effects of interleukin-6 antagonists for patients hospitalized with COVID-197. Due to the dynamic nature of the pandemic, and the need to align with World Health Organization guideline publication ${ }^{8}$, timely and robust synthesis of the accumulating trial results was vital. First, representatives from all eligible trials were invited to weekly meetings to develop the protocol and analytical approaches before trial results were known. Then, during a set period, and again before most trials were published, we collected highly detailed summary data, employing rigorous procedures for cross-checking of baseline and outcome information with available trial reports. This allowed us to query and rectify any discrepancies with the trialists. Ultimately, we obtained results for 27 of 29 trials, relating to over $95 \%$ of participants randomized (at that time). As only 9 of the 27 trials had reported results at the time of the PMA publication ${ }^{7}$, an equivalent meta-analysis based on published summary data would have been very limited, or would have been delayed until more trials had reported results. The FAME approach to $\mathrm{PMA}^{4}$ has also been used to provide timely evaluations of the effects of treatments for prostate cancer (example, ref. ${ }^{9}$ ).

Lawrence and colleagues recommend that "meta-analysts who study interventions for COVID-19 should request and personally review IPD in all cases"'. Although we agree that access to IPD from COVID-19 related trials is the ideal, even if trialists were to "immediately follow best-practice guidelines and upload anonymized IPD" ${ }^{\prime \prime}$ after publishing trial results, issues remain. Accessing IPD from different data-sharing platforms, with variable modes of access, can be a slow process, and thereafter, the datasets can be limited or heavily redacted (i.e., "careful anonymization" ${ }^{1}$ ) in order to protect participant privacy ${ }^{10}$. Obtaining IPD direct from investigators can also be a lengthy process, due to the increasing complexity of data-sharing agreements and prolonged negotiations between legal teams ${ }^{10}$, and the time taken to prepare IPD. Also, once the IPD are shared, standardizing and checking IPD requires substantial expertise and resources within the review team ${ }^{6}$. Thus, although the benefits of IPD meta-analyses are considerable, sharing of IPD is not yet widespread or streamlined enough to allow timely evidence synthesis, which is paramount in the context of a global pandemic. In fact, if we had requested IPD for the PMA of interleukin-6 antagonists in COVID-19, we suspect that by the time of publication of this Correspondence in early 2022, we would still not have collected sufficient trial data - a delay that may have led to countless avoidable deaths.

There is a distinct possibility that a "study for which authors are not able or not willing to provide suitably anonymized IPD" could "be considered at high risk of bias...or excluded" ${ }^{\prime}$. However, there may be legitimate reasons for non-provision of IPD, and excluding evidence in this way, or labeling it as having a high risk of bias, may itself lead to increased bias or a lack of generalizability. For example, if only trials carried out in high-income settings with sufficient infrastructure for immediate data 
sharing were included, valuable evidence from lower-income settings might be disregarded. By contrast, in a prospective and collaborative approach to evidence synthesis, a combination of systematic data-verification processes and close interactions with trialists ${ }^{4}$ vastly reduces the possibility of including fraudulent data and reduces the burden of data preparation for trial teams.

In conclusion, we commend Lawrence and colleagues for their important work identifying questionable and fraudulent clinical trial data, and we also call for change. However, recognizing that access to substantial and standardized IPD is still some way off, we believe that in the interim, adopting a prospective and collaborative approach to meta-analyses of summary data can provide timely, thorough and reliable evidence.
Peter J. Godolphin (D) 1,2四,

Ewelina Rogozińska,2, David J. Fisher,

Claire L. Vale ${ }^{1}$ and Jayne F. Tierney ${ }^{1}$

${ }^{1}$ MRC Clinical Trials Unit at University College

London, Institute of Clinical Trials and Methodology,

London, UK. ${ }^{2}$ These authors contributed equally:

Peter J. Godolphin, Ewelina Rogozińska.

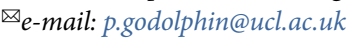

Published online: 10 February 2022

https://doi.org/10.1038/s41591-021-01675-1

\section{References}

1. Lawrence, J. M., Meyerowitz-Katz, G., Heathers, J. A. J., Brown, N. J. L. \& Sheldrick, K. A. Nat. Med. 27, 1853-1854 (2021).

2. Kwon, D. Nature 581, 130-131 (2020).

3. Ledford, H. et al. Nature $\mathbf{5 8 2}, 160$ (2020).

4. Tierney, J. F. et al. PLoS Med. 18, el003629 (2021).

5. Page, M. J. et al. PLoS Med. 13, el002028 (2016).

6. Tierney, J.F. et al. in Individual Participant Data Meta-Analysis: A Handbook for Healthcare Research (eds. Riley, R.D., Tierney, J.F. \& Stewart, L.A.) 9-19 (Wiley, 2021)
7. Shankar-Hari, M. et al. J. Am. Med. Assoc. 326, 499-518 (2021).

8. Rochwerg, B. et al. Br. Med. J. 370, m3379 (2020).

9. Vale, C. L. et al. Lancet 396, 1422-1431 (2020).

10. Rydzewska, L.H.M. et al. Trials (in the press).

\section{Acknowledgements}

E.R., D.J.F., C.L.V. and J.F.T. are supported by the UK Medical Research Council (grant MC_UU_00004/06). D.J.F. and P.J.G. are supported in part by Prostate Cancer UK (grant RIA 16-ST2-020). P.J.G. is supported in part by a UK National Institute for Health Research Development and Skills Enhancement Award (grant NIHR301653). The funders had no role in study design, data collection and analysis, decision to publish, or preparation of the manuscript.

\section{Author contributions}

P.J.G. and E.R. conceived of the idea of the manuscript and wrote the first draft. All authors commented on draft versions of the manuscript for important intellectual content and approved the final version.

\section{Non-Invasive Biomarkers of Nonalcoholic Steatohepatitis: the FNIH NIMBLE project}

To the Editor - Nonalcoholic fatty liver disease (NAFLD) affects approximately one-quarter of the global adult population ${ }^{1}$. A subset of affected individuals worldwide have nonalcoholic steatohepatitis (NASH), a more progressive form of the disease that has a higher risk of advancing to cirrhosis and end-stage liver disease (ESLD). Given the enormous number of afflicted patients, identification of the subset at risk of disease progression is critically important for efficient therapy allocation.

NASH patients with fibrosis stage 2 or higher have elevated all-cause and liver-related mortality ${ }^{2}$, and those with high disease activity scores are at greater risk of fibrosis progression ${ }^{3}$. These at-risk $\mathrm{NASH}$ patients are the target population for therapeutic intervention beyond lifestyle improvement. Histopathological assessment is the current reference standard for diagnosis, risk stratification and therapeutic efficacy evaluation for NAFLD. Unfortunately, liver biopsy for histological assessment carries risks, including even rare mortality. Histopathological assessment is also susceptible to sampling error and intra- and inter-reader variability. These drawbacks of liver biopsy have limited biopsy-based assessment in routine practice and pose challenges in clinical trial design and interpretation. There is therefore a need for reliable non-invasive tools for the diagnosis, risk stratification and monitoring of the course of NAFLD.

The integration of non-invasive tools into routine practice and in trial design requires both acceptance by the scientific community and, ideally, regulatory endorsement. The latter is particularly relevant to the use of non-invasive tools for defining trial populations and monitoring the course of disease with or without therapeutic intervention. An important approval pathway for non-invasive tools by the US Food and Drug Administration (FDA) is the Biomarker Qualification Program (BQP) ${ }^{4}$, which allows multiple stakeholders to come together to establish the utility of a non-invasive tool for its specific intended use. Substantial resources are needed for planning, data acquisition and analysis to meet the evidentiary burden for a full qualification package. This often requires a collaborative approach, and the likelihood of success can be maximized by pooling resources and expertise in a multi-stakeholder publicprivate partnership ${ }^{4}$.

The NIMBLE (Non-Invasive Biomarkers of Metabolic Liver Disease) consortium is a comprehensive, multi-year, pre-competitive public-private partnership collaboration conducted under the auspices of the Foundation for the NIH (FNIH) Biomarkers
Consortium. The Biomarkers Consortium brings together partners from academia, industry, regulatory bodies and nonprofit organizations to identify, develop and qualify potential biomarkers to improve drug development and regulatory decision-making 5 . The Metabolic Disorders Steering Committee and the Biomarker Consortium Executive Committee provide oversight for NIMBLE. NIMBLE is led by academic and industry co-chairs for the entire project and has two workstreams, for circulating biomarkers and for imaging biomarkers. NIMBLE is supported by a project team whose membership includes researchers from academia and industry and designated members from the FDA, who advise on project strategy without participating in the approval process for non-invasive tools. A central goal of NIMBLE is to systematically address and eliminate gaps in the existing scientific literature, thereby advancing FDA BQP qualification of one or more biomarkers for diagnosis and disease monitoring.

NIMBLE is structured as a two-stage project (Fig. 1). In stage 1, which is currently underway, the circulating biomarkers workstream will evaluate select biomarker panels for their ability to diagnose NASH and its activity, fibrosis stage or the presence of at-risk NASH (a composite including the 